\title{
Über das Verhalten des dielektrischen Verlustfaktors von Naturholz im großen Frequenzbereich
}

\author{
Kröner, Karl \\ Pungs, Leo
}

Veröffentlicht in:

Abhandlungen der Braunschweigischen Wissenschaftlichen Gesellschaft Band 4, 1952, S. 127-145

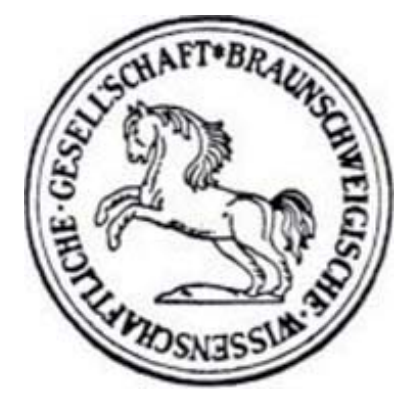

Friedr. Vieweg \& Sohn, Braunschweig 


\section{Utber das Verhalten des dielektrischen Verlustfaktors von Naturholz im großen Frequenzbereich \\ Von Karl Kröner und Leo Pungs}

Mit 12 Abbildungen

Abstract: The former research on the dielectric anisotropy of wood in the frequency range of $3 \cdot 10^{2} \ldots 10^{9} \mathrm{cps}$ has been extended by the measurement of the dielectric losses. The anisotropy originates in the macroscopical structure; the maximum of the loss-factor tg $\delta$ at rather high frequencies is explained by the dipole-character of cellulose. The effect of water comtained in the wood on the dielectric properties at higher frequencies is explained by the influence which the water molecules exercise on the vibration of the dipolegroups of the cellulcse. At low frequencies the influence of moisture comes from the inhomogenity corresponding to Wagner's theory of dielectric losses.

\section{Allgemeines}

In einer früheren Arbeit (Abhandlungen der B.W.G. Bd. I, S. 101) haben wir das Verhalten der Dielektrizitätskonstante von Naturhölzern im großen Frequenzbereich von $3 \cdot 10^{2}$ bis $1 \cdot 10^{9} \mathrm{~Hz}$ behandelt. In der vorliegenden Arbeit soll die Untersuchung auch auf den dielektrischen Verlustfaktor ausgedehnt werden.

Es wurden Holzproben mit Schnittrichtungen nach den 3 Symmetrieebenen, wie in der genannten Arbeit, untersucht. Nach dem in der Technik üblichen Verfahren bezeichnen wir den Verlustfaktor mit $\operatorname{tg} \delta$, wobei

$$
\operatorname{tg} \delta=\frac{\varepsilon^{\prime \prime}}{\varepsilon^{\prime}} \text { und } \varepsilon_{k}=\varepsilon^{\prime}+i \varepsilon^{\prime \prime}
$$

wenn $\varepsilon_{k}$ die komplexe Dielektrizitätskonstante bedeutet. Für die 3 Schnitte haben wir als Bezeichnungen:

$$
\begin{aligned}
& \operatorname{tg} \delta_{h}-\text { Hirnschnitt } \\
& \operatorname{tg} \delta_{t}-\text { Tangentialschnitt } \\
& \operatorname{tg} \delta_{r}-\text { Radialschnitt. }
\end{aligned}
$$

\section{Experimentelle Ergebnisse}

Die Methoden der Untersuchungen waren die gleichen wie in der früheren Arbeit. Alle diese Methoden erlauben, außer der reellen auch die imaginäre Komponente von $\varepsilon_{k}$ zu bestimmen. Für die Bestimmung bei sehr hohen Frequenzen $\left(10^{9} \mathrm{~Hz}\right)$ (im $\mathrm{cm}$ - und $\mathrm{dm}$-Wellengebiet) ist im Schrifttum auch das Verfahren der ummittelbaren Absorptionsmessung, analog den optischen Verfahren, vorgeschlagen worden $\left.{ }^{1}\right)^{13}$ ). Fo ist aber leicht zu zeigen, $\mathrm{daB}$ im Bereich der vorkommenden $\operatorname{tg} \delta$-Werte für Naturholz und bei den höchsten von uns angewandten Frequenzen die genaue Messung auf diesem Wege schwierig ist. Bei Vernachlässigung der Reflexion gilt für die Feldatärke $E$ 
nach der Absorption, wenn $E_{0}$ die Ausgangsfeldstärke ist:

$$
E=E_{0} \cdot \exp \left(-\frac{4 \pi k d}{\lambda}\right)
$$

Absorptionskoeffizient $k=\varkappa \sqrt{\varepsilon^{\prime}} ; d-$ Schichtdicke in $\mathrm{cm}, \lambda-$ Wellenlänge in $\mathrm{cm}$.

Zwischen $\approx$ und $\operatorname{tg} \delta$ besteht die Beziehung

$$
\operatorname{tg} \delta=\frac{2 x}{1-x^{2}}, \quad \text { daraus } \quad x=-\frac{1}{\operatorname{tg} \delta}+\sqrt{1+\frac{1}{\operatorname{tg}^{2} \delta}} .
$$

Berechnet man beispielsweise die Feldstärke $E$ für $d=3 \mathrm{~cm}, \lambda=30 \mathrm{~cm}$, $\varepsilon^{\prime}=2$ und $\operatorname{tg} \delta=300 \cdot 10^{-4}$, also für Daten, die ungefähr der unteren Grenze der gemessenen Werte bei Holz entsprechen, so erhält man nur $3 \%$ Unterschied gegenüber $\boldsymbol{E}_{0}$. Diese Betrachtung ist deshalb von einigem Interesse, weil man im Schrifttum auf Grund von Absorptionsversuchen die Angabe findet, daß die Absorption bei Holz sehr klein ist, während ein tg $\delta$ von der Größenordnung $300 \cdot 10^{-4}$ im technischen Sinne schon als beträchtlich angesehen wird. Für die Messungen bei sehr hohen Frequenzen haben wir das Verfahren der Meßleitung beibehalten.

Die Messungen sind für die gleichen Holzarten und unter den gleichen Bedingungen ausgeführt worden, wie sie in der früheren Arbeit für die Bestimmung der DK angenommen wurden.

\section{Holz im Darrzustande}

Die Abb. 1 zeigt die Ergebnisse der Messung von $\operatorname{tg} \delta$ in Abhängigkeit von der Frequenz (im logarithmischen Maßstab aufgetragen) für Fichtenholz in

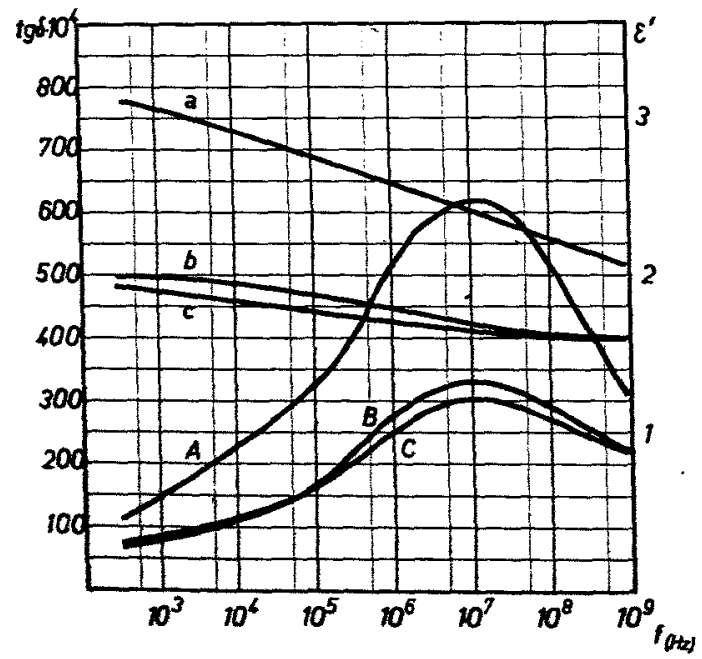

Abb. I. Fichte, DK und tg in Abhäglgkeit von der Frequens $f$ in Darrzugtand $\left(t=20^{\circ}\right)$

DK: a-Eirnsehnitt, b-radial, c-tangential,

t8 8: A-Fimsehnitt, B-redial, C-tangential. 
den 3 Schnitten. Die Abb. 2 gibt die gleichen Werte für Rotbuchenholz. Alle Untersuchungen sind im Darrzustande bei einer Temperatur von $20^{\circ}$

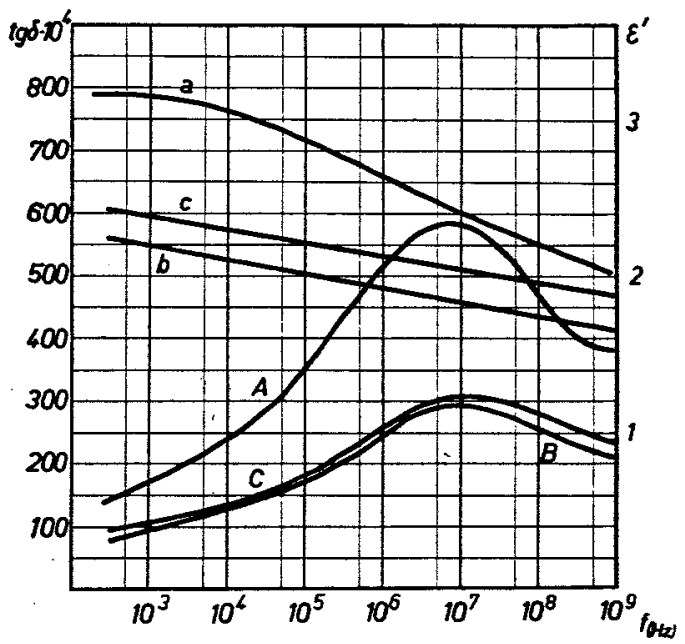

Abb. 2. Rotbuche, DK und tg $\delta$ in Abhängigkeit von der Frequenz im Darrzustand $\left(t=20^{\circ}\right)$

DK: $a$-Hirnschnitt, $b$-radial, $c$-tangential,

tg $\delta$ : $A$-Hirnschnitt, $B$-radial, $C$-tangential.

durchgeführt worden. Das Holz ist wie in der früheren Arbeit getrocknet worden. Der Vollständigkeit halber sind auch die DK-Werte mit eingetragen. Zum Vergleich führen wir wieder die Werte für Eichenholz an, für die gleichen Frequenzen wie auf Seite 102 der früheren Arbeit

\begin{tabular}{llll}
$f \mathrm{~Hz}$ & \multicolumn{1}{c}{$\operatorname{tg} \delta_{h}$} & \multicolumn{1}{c}{$\operatorname{tg} \delta_{t}$} & $\operatorname{tg} \delta_{r}$ \\
$3 \cdot 10^{2}$ & $140 \cdot 10^{-4}$ & $110 \cdot 10^{-4}$ & $110 \cdot 10^{-4}$ \\
$1 \cdot 10^{8}$ & $375 \cdot$ & $310 \cdot$ & $.250 \cdot$
\end{tabular}

Die Ergebnisse lassen sich wie folgt zusammenfassen:

a) Die Abhängigkeit des tg $\delta$ von der Frequenz für die beiden Holzarten zeigt einen charakteristischen Verlauf mit einem breiten Maximum, das bei den versehiedenen Kurven zwischen $5 \cdot 10^{6}$ und $10^{7} \mathrm{~Hz}$ liegt ${ }^{3}$ ). Auch für Eichenholz erhielten wir ein Maximum im gleichen Frequenzbereich.

b) Die Lage des Maximums wird durch die Schnittrichtung wenig beeinfluBt. Die gröBten Werte des tg $\delta$ erhält man immer für den Hirnschnitt. Für den Radial- und Tangentialschnitt ergeben sich nur kleinere Unterschiede. Diese Verhältnisse entsprechen sehr annähernd denen bei der DK.

\section{Holz mit höherem Wassergehall}

Wir erweiterten unsere Untersuchungen auf feuchte Holzproben, die Ergebnisse sind in der $\mathrm{Abb}$. 3 für Fiehte und $\mathrm{Abb}$. 4 für Rotbuche dargestellt, und zwar dieses Mal nur für den Hirnschnitt. Als Parameter sind die prozen- 
tualen Gewichtserhöhungen durch die Wasseraufnahme angegeben, die wie üblich auf das Darrgewicht bezogen werden. Temperatur ist wieder $20^{\circ}$. Für Eiche erhielt man Kurven von ähnlichem Verlauf, die wir aber hier nicht wiedergeben wollen.

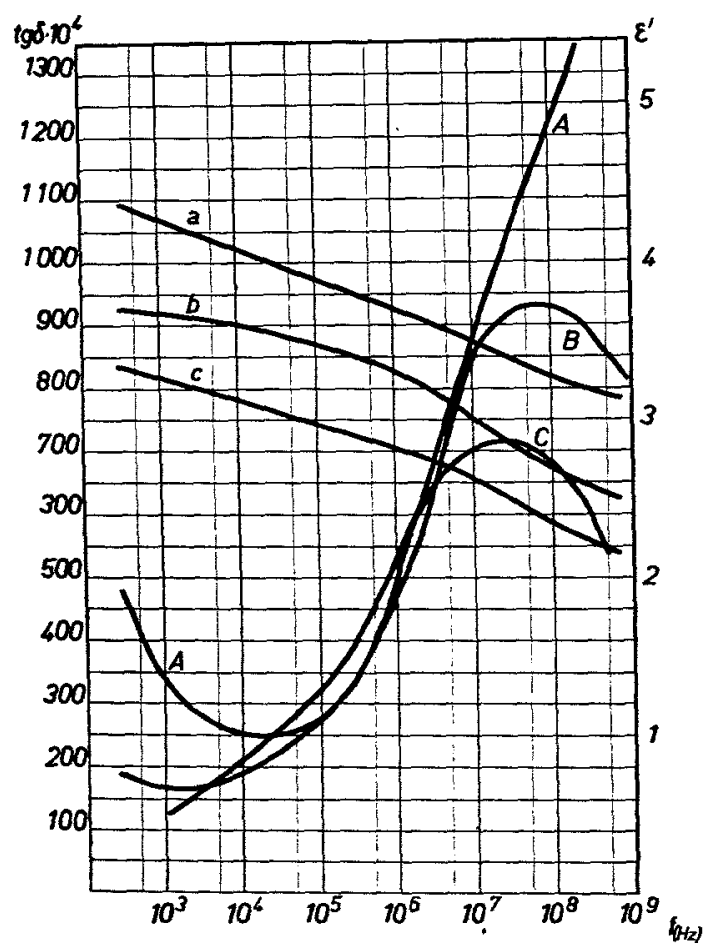

Abb. 3. Fichte, Hirnsehnitt, DK und $\mathrm{tg} \delta$ in Abhängigkeit von der Frequenz beliferschiedenen Feuchtigkeitsgraden $\left(\ell=20^{\circ}\right)$

$$
\begin{array}{rrrr}
\text { DK: } & a-11,8 \% & b-5,85 \% & c-2,65 \% \\
\text { tg } \delta: & A-11,8 \% & B-5,85 \% & C-2,65 \%
\end{array}
$$

Die Frgebnisse dieser Messungen sind:

a) Die DK steigt mit dem Wassergehalt. Die anomale Dispersion bleibt bestehen.

b) Im Bereich der mittleren Frequenzen $10^{4}-10^{6} \mathrm{~Hz}$ hängt der Verlanf des tg $\delta$ als Funktion der Frequenz nur wenig vom Wassergehalt ab, soweit er in den gegebenen Grenzen bleibt.

c) Bei Frequenzen unterhalb $10^{4} \mathrm{~Hz}$ zeigt der Verlustfaktor, insbesondere bei höherem Wassergehalt, einen starken Anstieg bei fallender Frequenz.

d) Im Frequenzgebiet über $10^{*} \mathrm{~Hz}$ haben wir zunächst eine Steigerung des tg $\delta$ und eine Verlagerung des Maximums zu höheren Frequenzen. Bei größerem Wassergehalt steigt der tgd mit der Frequenz sehr schnell an, 
ohne daß in dem untersuchten Gebiet eine Maximumbildung festgestellt werden konnte.

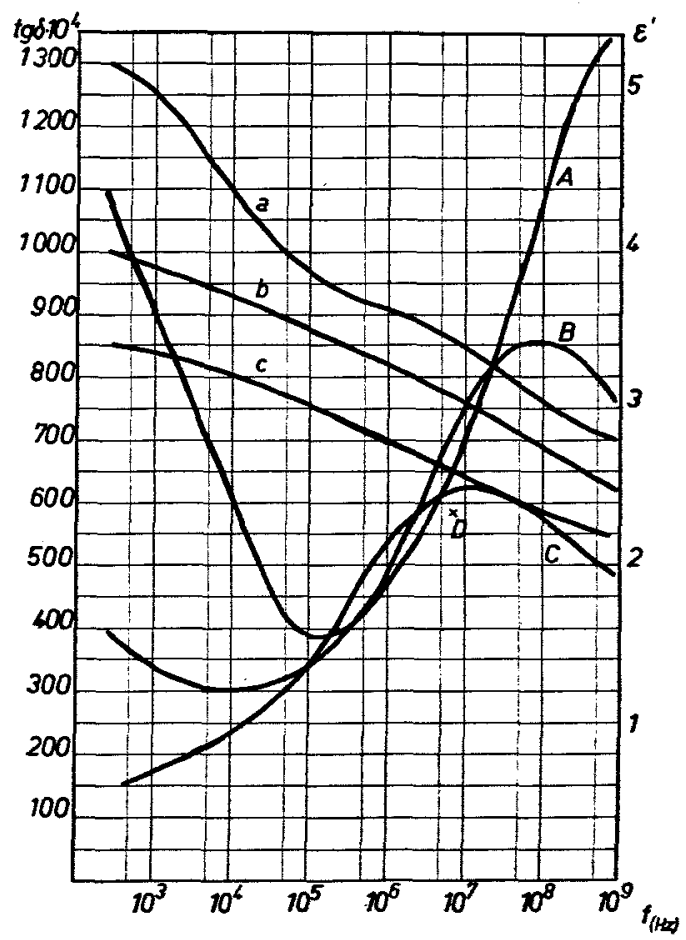

Abb. 4. Rotbuche, Hirnschnitt, $\mathrm{DK}$ und $\mathrm{tg} \delta$ in Abhängigkeit von der Frequenz bei verschiedenen Feuchtigkeitsgraden $\left(t=20^{\circ}\right)$

$$
\begin{array}{lllc}
\text { DK: } & a-12 \% & b-6,65 \% & c-2,5 \% \\
\text { tg } \delta: & A-12 \% & B-6,65 \% & C-2,5 * \%
\end{array}
$$

Punkt $D$-Maximalwert für tg $\delta$ bei Darrzustand (Abb. 2 ).

\section{Verhalten bei tiefen Frequenzen}

Der starke Anstieg des $\operatorname{tg} \delta$ bei tiefen Frequenzen für feuchtes Holz gab uns die Veranlassung, die. Verhältnisse in dem Frequenzbereich von 33 bis $10^{4} \mathrm{~Hz}$ noch gesondert zu untersuchen. Die Frgebnisse sind anf den $A b b .5$ und 6 gegeben. Sie beziehen sich auf Rotbuche im Tangentialschnitt bei $20^{\circ} \mathrm{C}$.

a) Während bei kleinem Wassergehalt $(<4 \%)$ die DK, wie im Darrzustande, kaum eine merkbare Dispersion zeigte, war bei höherem Wassergehalt ein starkes Ansteigen der DK mit abfallender Frequenz zu beobrohten.

b) Ein ähnliches Verhalten zeigt auch der Verlustfaktor in diesem Frequenzbereich. Bei $f=33 \mathrm{~Hz}$ wurden sehr hohe Werte des $\operatorname{tg} \delta$ bis über $0,6 \mathrm{er}$ reicht. 


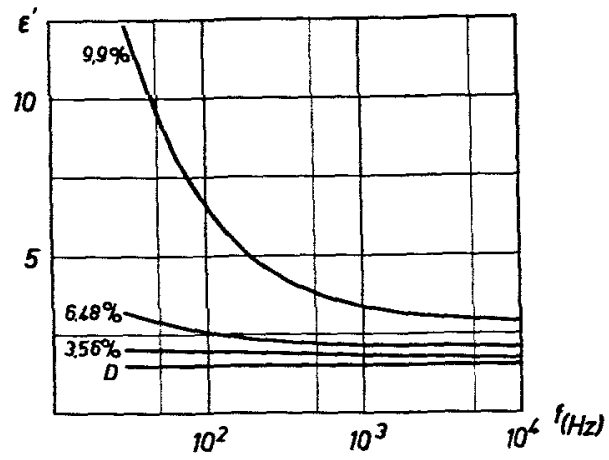

Abb. 5. Rotbuche, Tangentialschnitt, DK in Abhängigkeit von der Frequenz für tiefe Frequenzen bei $t=20^{\circ}$ und verschiedenen Feuchtigkeitsgraden (a. d. Kurven angegeben).

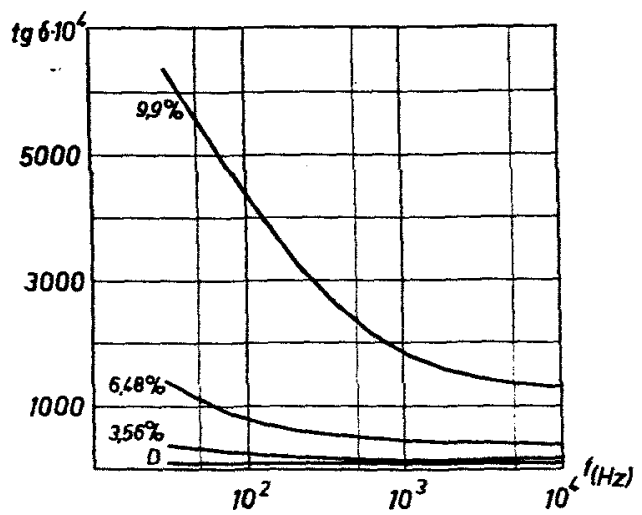

Abb. 6. Rotbughe, Tangentialschnitt, $\operatorname{tg} \delta$ in Abhs̈ngigkeit von der Frequenz fïr tiefe Frequenzen bel $t=20^{\circ}$ und verschiedenen Fenchtigkeitagraden ( . d. Kurven angegeben).

\section{Versuch einer theoretischen Deatung der Ergebnisse}

1. Anisolropie des Verlustfaktors im Darrzustande

Bei der Untersuchung der DK haben wir, soweit es die komplizierten Verhältnisse bei Naturholz erlaubten, die Anisotropie durch den makroskopischen Bau des Holzes zu erklären versucht. Für die Anisotropie des tg $\delta$ oder der imaginären Komponente der DK müssen die gleichen Ursachen angenommen werden. Der Vergleich der Werte von $\varepsilon^{\prime}$ und tg $\delta$ für verschiedene Schnittrichtungen geben schon einen Hinweis auf ein ähnliches Verhalten beider Werte.

Die von uns bei dex DK für dos Mischdielektrikum Zellsubstanz + Luft angewendete Lichtenecker-Rothersche Potenzformel ist von Büchner auch ouf den tgঠ auggedehnt worden, indem er

$$
\varepsilon=\varepsilon^{\prime}+i \varepsilon^{\prime \prime}
$$


setzte und diesen Wert in die Formeln für $\varepsilon$ einführte. Für kleine Werte von $\operatorname{tg} \delta(<0,1)$, wie sie hier nur in Frage kommen, erhält man die vereinfachte Formel

$$
\operatorname{tg} \delta=\frac{\sum_{n} \vartheta_{n}\left(\varepsilon_{n}^{\prime}\right)^{k} \operatorname{tg} \delta_{n}}{\sum_{n} \vartheta_{n}\left(\varepsilon_{n}^{\prime}\right)^{k}}
$$

$\left(\vartheta_{n}\right.$ - Volumina der Mischsubstanzen) .

Wir nehmen in grober Annäherung, wie früher, die Parallelschaltung für den Hirnschnitt $(k=+1)$ und Reihenschaltung für den Tangentialschnitt $(k=-1)$ an, wobei wir wieder als Schichten ein Mischdielektrikum zweiter Ordnung mit annähernd gleichmäßiger Verteilung beider Komponenten zugrunde legen. Das Mischdielektrikum erster Ordnung entsteht dann durch Parallel- oder Reihenanordnung dieser Schichten mit Luftschichten.

Wir erhalten bei $\varepsilon_{2}^{\prime}=1 ; \operatorname{tg} \delta_{2}=0$ (Laft)

$$
\operatorname{tg} \delta_{h}=\frac{\vartheta_{1} \varepsilon_{1}^{\prime} \operatorname{tg} \delta_{1}}{\vartheta_{1} \varepsilon_{1}^{\prime}+\vartheta_{2}} ; \quad \operatorname{tg} \delta_{t}=\frac{\frac{\vartheta_{1}}{\varepsilon_{1}^{\prime}} \cdot \operatorname{tg} \delta_{1}}{\frac{\vartheta_{1}}{\varepsilon_{1}^{\prime}}+\vartheta_{2}} .
$$

$\operatorname{tg} \delta_{1}$ ist der Verlustfaktor des Mischkörpers zweiter Ordnung, der zunächst unbekannt ist. Dagegen können wir $\varepsilon_{1}^{\prime}$ aus den $A b b .2$ und 3 unserer früheren Arbeit entnehmen. Um unsere Annahme weiter zu verfolgen, führen wir das Verhältnis

$$
v=\frac{\operatorname{tg} \delta_{h}}{\operatorname{tg} \delta_{l}}
$$

ein, das von $\operatorname{tg} \delta_{1}$ unabhängig ist. Wir setzen

und erhalten

$$
\gamma=\frac{\theta_{1}}{\theta_{2}}
$$

$$
v=\frac{\frac{\vartheta_{1}}{\vartheta_{2}}+\varepsilon_{1}^{\prime}}{\frac{\vartheta_{1}}{\vartheta_{2}}+\frac{1}{\varepsilon_{1}^{\prime}}}=\frac{\gamma+\varepsilon_{1}^{\prime}}{\gamma+\frac{1}{\varepsilon_{1}^{\prime}}}
$$

Für Fichtenholz entnehmen wir aus unserer früheren Rechnung für den Maximalwert des $\operatorname{tg} \delta_{\mathrm{h}}$ bei $f=10^{7} \mathrm{~Hz}$ die $\mathrm{DK} \varepsilon_{1}^{\prime}=3,5$. Wir erhalten weiter

$$
\begin{aligned}
& \gamma=\frac{0,58}{0,42}=1,38 \\
& v=\frac{1,38+3,5}{1,38+0,29}=2,8 .
\end{aligned}
$$

Der aus den Kurven entnommene Wert für $v$ bei $f=10^{7} \mathrm{~Hz}$ ist 2,06. Die thereinstimmung ist nur gröBenordnungsmäBig. Sie spricht aber nicht gegen eine Erklärung der Anisotropie durch die makroskopische Struktur. Es ist natürich, daB bei dem komplizierten Bau des Naturhalzes die schematische Annahme der Parallel- und Reihensehaltung nur eine enste Annäherung an die wirklichen Verhältnisse darstellte. Für eine solche ist die Ubereinstimmung genügend. Es mäBte bei weiterer Verfolgang noch eine Korrektur an der 
Konstanten $k$ angebracht werden, auf die wir hier aber nicht eingehen wollen. Es folgt aus diesen Betrachtungen, daß, ebenso wie bei der DK, die Anisotropie des tg $\delta$ mit großer Wahrscheinlichkeit aus der makroskopischen Struktur des Holzes zu erklären ist.

\section{Dispersion des Verlustfaktors im Darrzustande}

Das Auftreten eines, wenn auch sehr flachen Maximums des $\operatorname{tg} \delta$ in einem bestimmten Frequenzbereich ist für alle Holzarten charakteristisch. Wir haben außer den Untersuchungen, deren Ergebnisse in den Abb. 2 und 3 gegeben sind, auch Messungen an anderen Holzprodukten wie Holzmehl und Zellstoff ausgeführt und geben in der folgenden Tabelle die Frequenzen an, die dem Maximalwert des $\operatorname{tg} \delta$ entsprechen:

$\begin{array}{lr} & f_{0}\left(H_{z}\right) \\ \text { Rotbuche, Hirnschnitt } & 0,7 \cdot 10^{7} \\ \text { Fichte, Hirn } & 1 \cdot 10^{7} \\ , \quad \text { tangential } & 1 \cdot 10^{7} \\ \text { Eiche, Hirn } & 1 \cdot 10^{7} \\ \text { tangential } & 0,5 \cdot 10^{7} \\ \text { Kieferholzmehl } & 1,2 \cdot 10^{7} \\ \text { Buchenholzmehl } & 1 \cdot 10^{7} \\ \text { Buchenholzzellstoff } & 1 \cdot 10^{7} \\ \text { [Zellon } & 1 \cdot 10^{7} \\ \end{array}$

Die Daten beziehen sich auf Mischkörper. Da aber die eine Komponente Luft ist, wird, wie wir später an einem besonderen Beispiel zeigen werden, wohl der Wert, nicht aber die Frequenz des Maximums wesentlich beeinflußt.

Wir erkennen, daß trotz der Verschiedenheiten der Grobstruktur, besonders auch bei dem Mehl (mit Teilchen von im Mittel 3,2 $\mu$ Ausdehnung), die Frequenz $f_{0}$ immer in der Nähe von $10^{7} \mathrm{~Hz}$ liegt. Die Abweichungen bleiben bei dem sehr flachen Verlauf des Maximums innerhalb der MeBgrenzen. Es muB also mit großer Wahrscheinlichkeit angenommen werden, daß die Verluste molekularer Art im Sinne der Debyeschen Dipoltheorie sind und durch die polaren Eigenschaften des Grundstoffes der Zellsubstanz, also in erster Linie der Zellulose, zu erklären sind. Auch die anomale Dispersion der DK wäre dann auf die gleiche Ursache zurückzufïhren, wie wir das in der früheren Arbeit schon erwähnt hatten. Die Wagnersche Inhomogenitätstheorie kann zur Deutung der Erscheinungen im hohen Frequenzbereich nicht herangezogen werden, weil dann eine wesentliche Änderung der Frequenz $f_{0}$ mit Veränderung der makroskopischen Struktur zu erwarten wäre. Außerdem müßte das Maximum für $\operatorname{tg} \delta$ bei wesentlich tieferen Frequenzen liegen (vgl. S. 140).

Es wäre natürlich von Interesse, den Verlanf des tg $\delta$ im großen Frequenzbereich für reine Zellulose zu kennen. Im Sebrifttum ${ }^{22}$ ) wird ein Maximum des $\operatorname{tg} \delta$ bei $f \approx 10^{6} \mathrm{~Hz}$ angegeben. In einer neueren Arbeit von $\mathrm{Henninger}{ }^{8}$ ), die sich eingehend mit den dielektrischen Eigenschaften der Zellulose beschäftigt, sind Kurven für den tg $\delta$ von Papierfaser in Abhängigkeit von der Frequenz bis $2 \cdot 10^{5} \mathrm{~Hz}$ enthalten, also nur für den unteren Teil des von uns 
untersuchten Bereichs. Die Messungen des Verlustfaktors der reinen Zellulose mit Hilfe eines Imprägnierungsverfahrens mit Stoffen gleicher DK sind bei hohen Frequenzen etwas schwierig und lagen auch nicht im Rahmen dieser Arbeit. Wir beabsichtigen, an anderer Stelle darauf zurückzukommen. Hier handelt es sich darum, festzustellen, ob der Verlauf der DK und insbesondere des tg $\delta$ beim Holz in erster Linie durch die polaren Eigenschaften der Zellulose und der weiteren Komponenten, der Holzpolyosen (Hemizellulosen) bedingt ist. Das läßt sich aber auch durch eine Untersuchung eines Mischdielektrikums Zellulose + Luft im gleichen Frequenzbereich, wie beim Holz, mit großer Wahrscheinlichkeit entscheiden. Natürlich wird hier der Wert des tg $\delta$ nur einen Teil desjenigen für reine Zellulose betragen, doch wird die Frequenzlage des Maximums nur wenig verschoben. Um diese Verschiebung abzuschätzen, nehmen wir für unseren Mischstoff Zellulose + Luft zunächst den Grenzfall für Reihenschaltung nach Gl. (2) an.

$$
\operatorname{tg} \delta^{\prime \prime}=\frac{\frac{\vartheta_{1}}{\varepsilon_{1}^{\prime}} \cdot \operatorname{tg} \delta_{1}}{\frac{\vartheta_{1}}{\varepsilon_{1}^{\prime}}+\vartheta_{2}}=\frac{1}{1+\frac{\varepsilon_{1}^{\prime}}{\gamma}} \operatorname{tg} \delta_{1} .
$$

Wir sehen also, daß der Verlauf von $\operatorname{tg} \delta^{\prime \prime}$ im Vergleich mit dem Verlauf von $\operatorname{tg} \delta_{1}$ durch die Abhängigkeit der DK $\varepsilon_{1}^{\prime}$ von der Frequenz und zwar im Bereich des Maximums bestimmt wird. Auf der Abb. 7 (Kurve $C$ u. B) ist die DK und

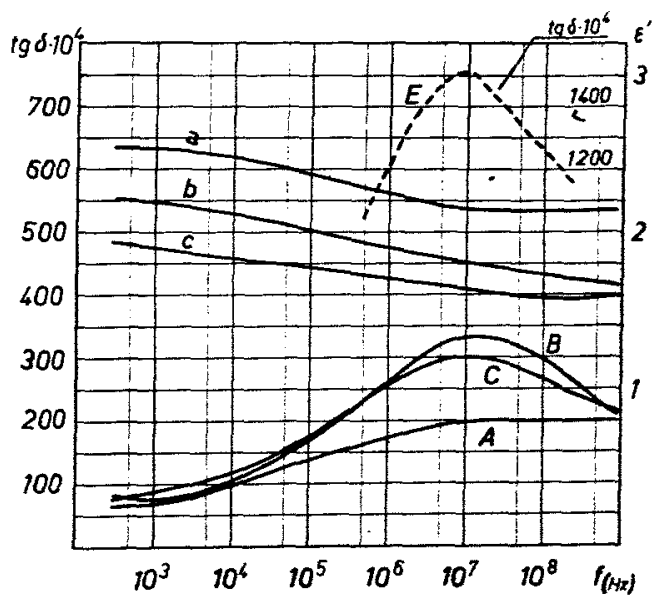

Abb. 7 . DK und tg $\delta$ in Abhängigkeit ron der Frequenz bei trockenen Stoffen and $20^{\circ} \mathrm{C}$

DK: $a$-Lignin, $b$-Zellulose + Luft, $c$-Fichte tangential

$\operatorname{tg} b: A^{-},, B *, \quad+, C-, "$,

$E$ tg $\delta$ fúr Zellulose ans dem Mischdielektrikum mit Reihenschaltung berechnet. Yabstab rechts.

der $\operatorname{tg} \delta^{\prime \prime}$ des Mischdielektrikums Zellulose + Luft aufgetragen, sowie die für Reihenschaltung berechneten Werte der reinen Zellulose $(B)$, wobei für $\gamma$ der ungünstigste Wert 1 angenommen worden ist. Wir sehen, daB trotz des großen Anwachsens des Wertes von $\operatorname{tg} \delta_{1}$ die Frequenz für das Maximum beinahe 
unverändert bleibt. Nimmt man Parallelschaltung an, so ist wieder für $\gamma=1$

$$
\operatorname{tg} \delta^{\prime}=\left(1+\frac{1}{\varepsilon_{1}^{\prime}}\right) \cdot \operatorname{tg} \delta_{1} .
$$

Der Faktor in der Klammer ändert sich bei gleicher Änderung von $\varepsilon_{1}^{\prime}$, noch langsamer als bei der Reihenschaltung, also wird auch hier das Maximum des reinen Stoffs in nächster Nähe des Maximums des Mischdielektrikums liegen. Der wirkliche Wert der Frequenz $f_{0}$ für reine Zellulose, der zwischen den beiden Grenzwerten der Reihen- und Parallelschaltung liegt, wird also sehr nahe dem Werte des Mischdielektrikums entsprechen und $10^{7} \mathrm{~Hz}$ betragen $(\lambda=30 \mathrm{~m})$. Die entsprechende wahrscheinlichste Relaxationszeit beträgt dann

$$
\tau_{0}=\frac{1}{2 \pi f_{0}}=1,6 \cdot 10^{-8} \mathrm{~s} .
$$

Die Tatsache, daß die Naturhölzer und die Zellulosestoffe ein Maximum $\operatorname{des} \operatorname{tg} \delta$ bei einer Frequenz um $10^{7} \mathrm{~Hz}$ haben, läßt mit großer Wahrscheinlichkeit den Schlu $B$ zu, daß der Frequenzverlauf der DK und des $\operatorname{tg} \delta$ dieser Körper in erster Linie durch die polaren Eigenschaften der Zellulose bedingt ist. Dazu kommt noch der Einfluß der Holzpolyosen, die immerhin etwa 30\% der Zellsubstanz bilden können. Nach den Messungen muß entweder der Einfluß dieser Komponenten nicht groß sein oder die Relaxationszeiten müssen in den gleichen Frequenzbereich fallen wie bei der Zellulose. Das Lignin, als weitere Komponente des Holzes, zeigt innerhalb des interessierenden Frequenzbereiches um das Maximum der Zellulose nur geringe Änderungen des tg $\delta$ (Abb. 7). Es wird also die Lage des Maximums kaum beeinflussen. Die Harzbestandteile haben relativ kleinen Verlustfaktor in diesem Bereich und wegen ihrer geringen Menge kaum einen Einfluß auf den Verlauf des Verlustfaktors des Holzes.

Vergleicht man den Verlauf $\operatorname{des} \operatorname{tg} \delta=\varphi(f)$ bei Holz mit dem Verlauf bei polaren Flüssigkeiten definierter chemischer Zusammensetzung, insbesondere wenn es sich um eine Lösung in einem nicht polaren Stoff handelt, so fällt das schon erwähnte sehr flache Maximum auf. Auch die DK zeigt keine ausgesprochene Sprungstelle in der Nähe des Maximums für tg $\delta$.

Für die einfachen Verhältnisse bei polaren Molekülen mit einer einzigen Relaxationszeit gelten die bekannten Debyeschen Beziehungen.

$$
\begin{aligned}
& \varepsilon^{\prime}=\varepsilon_{0}+\frac{\varepsilon_{8}-\varepsilon_{0}}{1+\left(\frac{\varepsilon_{s}+2}{\varepsilon_{0}+2}\right) \omega^{2} \tau^{2}} \\
& \varepsilon^{\prime \prime}=\left(\varepsilon_{3}-\varepsilon_{0}\right) \cdot \frac{\frac{\varepsilon_{2}+2}{\varepsilon_{s}+2} \cdot \omega \tau}{1+\left(\frac{\varepsilon_{2}+2}{\varepsilon_{0}+2}\right) \omega^{2} \tau^{2}}
\end{aligned}
$$

Es bezeichnet hier $\varepsilon_{0}$ wie oben die DK für sehr hohe Frequenzen, $\varepsilon_{s}$ die statische DK für $f=0$.

Diese Formeln ergeben eine relativ schmale $\operatorname{tg} \delta=f(\omega)$-Kurve. Die Verbreiterung bei Zellulose, die ein hochpolymerer Stoff ist, und bei Naturhola kann entweder aus der Kopplung der einzelnen Dipole oder aus verschiedenen verteilten Relaxationszeiten der schwingenden Elemente erklärt werden. Insbesondere die zweite Uraache ist für Zellulose sehr naheliegend, da sie keinen 
Stoff mit einheitlichem Polymerisationsgrad darstellt. Sie besteht also aus Molekülen mit schwingenden Gruppen verschiedener Relaxationszeit. Aber auch die erste Ursache, die Kopplung der Dipole, ist vorhanden, und beides wirkt sich in der Weise aus, daß verschiedene nach einer bestimmten Funktion verteilte Relaxationszeiten auftreten. Dieser Fall ist von $K$. W. Wagner eingehend behandelt worden. Wenn auch die Wagnerschen Betrachtungen von der Theorie der dielektrischen Nachwirkung bei festen Stoffen ausgehen, so lassen sie sich auch auf die Vorgänge in Dipolstoffen übertragen. Nach dem Vorgange von Pellat und $\mathrm{Sch}$ weidle ${ }^{19}$ ) wird für den Verlauf der dielektrischen Nachwirkung im konstanten Felde eine exponentielle Funktion angenommen:

$$
\Psi(t)=\left(\frac{k}{\tau}\right) \cdot \exp \left(-\frac{t}{\tau}\right) .
$$

$\tau$ ist die Relaxationszeit, in der die Ladung auf $1 / e$ gesunken ist, $k=$ eine Konstante.

Auch De by e erhält den gleichen Exponential-Ausdruck für diese Funktion. Als Relaxationszeit definiert er die Zeit, in der der veränderliche Teil der Verteilungsfunktion anf den $1 / e$-Teil gesunken ist. Das bedeutet, daß die durch das Feld geordneten Moleküle in dieser Zeit zum Teil in den ungeordneten Zustand zurückfallen, wenn das Feld verschwindet. Die Grundgleichung für den Strom lautet dann:

$$
i_{a}=C \int_{-\infty}^{t_{1}} \frac{d U(t)}{d t} \cdot \Psi\left(t_{1}-t\right) d t
$$

wo $C$ die sog. geometrische Kapazität bedeutet. Führt man den Ausdruck (7) für $\Psi(t)$ ein und setzt für $U(t)=U$ sin $\omega t$, so erhält man nach Zerlegung des Stromes in einen reellen und einen imaginären Teil für die entsprechenden Komponenten der DK die bekannten Ausdrücke:

$$
\begin{aligned}
\varepsilon^{\prime} & =\varepsilon_{0}\left[1+k /\left(1+w^{2} \tau^{2}\right)\right] \\
\varepsilon^{\prime \prime} & =\varepsilon_{0} k w \tau /\left(1+\omega^{2} \tau^{2}\right)
\end{aligned}
$$

Das gilt für den Fall, daß alle Dipole die gleiche Relaxationszeit haben. Sind Gruppen von Dipolen mit verschiedenen Relaxationszeiten $\tau_{1}, \tau_{2}, \ldots, \tau_{n}$ vorhanden, wobei die Zahl der Dipole jeder Gruppe in der Raumeinheit $k_{1}, k_{2}, \ldots, k_{n}$ beträgt, so erhält man

$$
\Psi(t)=\sum_{n} \frac{k_{n}}{\tau_{n}} \cdot \exp \left(-\frac{t}{\tau_{n}}\right)
$$

und beim Ubergang auf eine unendliche Zahl

$$
\Psi(t)=\int_{0}^{\infty} \frac{k(\tau)}{\tau} \cdot \exp \left(-\frac{t}{\tau}\right) d t
$$

wobei $k(\tau)$ die Verteilungsfunktion für die Relaxationszeit ist. Für diese Verteilungsfunktion nimmt nun Wagner ${ }^{23}$ ) die Gleichung:

$$
k(\tau) d \tau=\frac{k b}{\sqrt{\pi}} \cdot \exp \left(-b^{2} z^{2}\right) d z
$$


an, wie sie von Wiechert in der Theorie der elastischen Nachwirkung vorgeschlagen worden ist. Wegen der näheren Begründung sei auf die Arbeit von Wagner verwiesen. Es bedeutet

$$
z=\ln \frac{\tau}{\tau_{0}}
$$

wenn $\tau_{0}$ der wahrscheinlichste Wert der Relaxationszeit $\tau$ ist, um die sich die anderen Werte ordnen. Die Konstante $b$ bestimmt die Dichte der Verteilung. Bei $b \rightarrow 0$ wird die Verteilungskurve $k(\tau)$ sehr breit. Bei $b \geqq 2$ ist sie schon sehr schmal. Führt man nun diese Verteilungsfunktion in die Gleichung (8) für $i_{a}$ ein, so erhält man nach einigen Zwischenrechnungen die Ausdrücke

$$
\begin{aligned}
& \varepsilon^{\prime}=\varepsilon_{0}\left[1+\frac{k b}{\sqrt{\pi}} \cdot \exp \left(-b^{2} z_{0}^{2}\right) \int_{0}^{\infty} \exp \left(-b^{2} u^{2}\right) \frac{\operatorname{CU}_{0}\left\{\left(2 b^{2} z_{0}-1\right) u\right.}{\mathfrak{C}_{0} j u} \cdot d u\right] \\
& \varepsilon^{\prime \prime}=\frac{\varepsilon_{0} k b}{\sqrt{\pi}} \cdot \exp \left(-b^{2} z_{0}^{2}\right) \int_{0}^{\infty} \exp \left(-b^{2} u^{2}\right) \frac{\mathbb{E}_{0 j} 2 b^{2} z_{0} u}{\operatorname{Evj} u} \cdot d u \\
& \text { wobei } \quad z_{0}=\ln \omega \tau_{0} \quad z=\ln \frac{\tau}{\tau_{0}} \\
& u=z+z_{0}=\ln \omega \tau
\end{aligned}
$$

Die Integrale lassen sich, einige Sonderfälle ausgenommen, nicht in geschlossener Form darstellen, und können nur mit Benutzung von Näherungsverfahren ausgewertet werden. Auf Grund der Wagnerschen Gleichung hat Yager ${ }^{24}$ ) Tabellen und Kurven angegeben, mit deren Hilfe man $\varepsilon^{\prime}$ und $\varepsilon^{\prime \prime}$ als Funktion von $\omega=2 \pi f$ ermitteln kann, wenn die Konstanten $b, k, \tau_{0}$ und $\varepsilon_{0}$ experimentell bestimmt sind. Für die Bestimmung dieser Konstanten auf Grund von Messungen von $\varepsilon^{\prime}$ und $\varepsilon^{\prime \prime}$ für 3 Frequenzen hat Yager ein graphisches Verfahren angegeben. Wegen der Einzelheiten des Verfahrens sei auf die Originalarbeit verwiesen*).

Wir haben das Verfahren für die Berechnung der DK und des $\operatorname{tg} \delta$ in Abhängigkeit von der Frequenz bei Fichtenholz im Hirnschnitt angewendet. Die Konstante $b$ wurde dabei mit dem Wert 0,15 ermittelt. Die Ergebnisse sind in Abb. 8 aufgetragen und mit den Messungen verglichen. Es ist auch die Berechnung für den Fall einer einzigen Relaxationszeit durchgeführt und ebenfalls zum Vergleich aufgetragen. Bei dem Verlauf der DK erkennen wir, daß der Sprung, der im Falle einer einzigen Relaxationszeit auftritt, bei einem verteilten $\tau$ undeutlich wird. Für die DK ist die Übereinstimmung der berechneten und gemessenen Werte ziemlich gut. Bei dem Verlustfaktor ist wohl der allgemeine Verlanf der berechneten und gemessenen Kurve im wesentlichen übereinstimmend, vor allem erkennt man das sehr flache Maximum. Die absoluten Werte zeigen jedoch eine größere Abweichung. Wenn man die uneinheitliche Struktur des Holzes bedenkt, so ist die Abweichung auch erklärlich. Es kann aber mit großer Wahrscheinlichkeit angenommen werden,

*) Andere Verfahren zur Berücksichtigung der Verteilung bei F.H.Müller a. Ch. Schmelzer ${ }^{15}$ ) S. 382. 
daß das dielektrische Verhalten des Naturholzes in erster Linie durch die polaren Eigenschaften der Zellulose als eines Stoffes mit verteilter Relaxations-

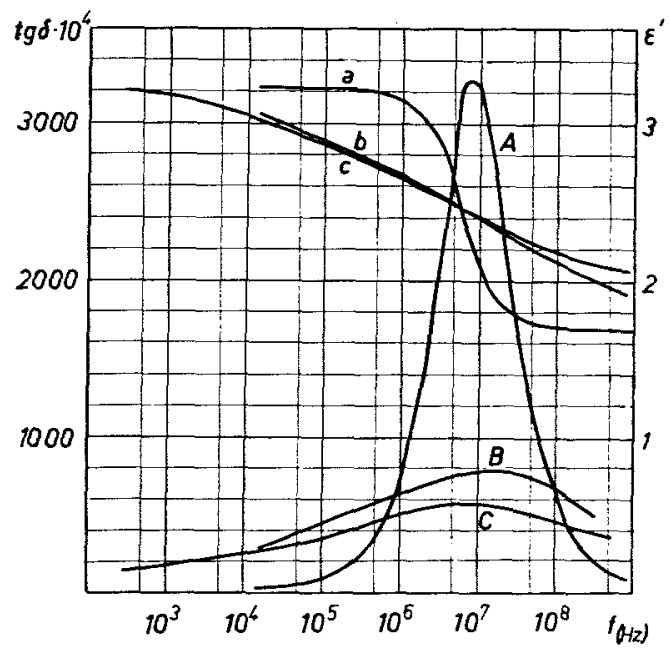

Abb. 8. DK und tg $\delta$ in Abhängigkeit von der Frequenz fïr Buchenholz-Hirn, gemessen und berechnet nach der Wagnerschen Verteilung der Relaxationszeit

DK : $a$ - nur eine Relaxationszeit, $b$ - naeh Wagner, $c$ - gemessen, $\operatorname{tg} \delta: A$ - wie $a, B$-wie $b, C$-wie $c$.

zeit der schwingenden Dipolgruppen bestimmt wird. Für die Holzpolyosen gilt das auf S. 139 Gesagte. Die Betrachtungen dieses Abschnittes werden auch durch die Untersuchungen an Glukose (S. 143, Abb. 12) gestützt.

\section{Verhalten bei Wassergehalt}

Die genauere Deutung dieses Verhaltens aus den an feuchtem Holz ausgeführten Untersuchungen ist wegen der komplizierten Struktur des Materials schwierig. Es soll an anderer Stelle auf Grund einer noch laufenden experimentellen Arbeit eine eingehendere Klärung dieser Sonderfrage versucht werden, bei der auch der EinfluB der Temperatur mit einbezogen werden soll.

Da man praktisch meistens mit einem Zustand des Naturholzes zu tun hat, der in bezug auf seinen Wassergehalt vom Darrzustande abweicht, sahen wir uns veranla $B$ t, auch den Einfluß des Wassergehaltes, wenn auch zunächst orientierend, in unsere Untersuchungen aufzunehmen. Auch wollen wir auf Grund des vorhandenen Materials versuchen, einige Aussagen zur Klärung der beobachteten Erscheinungen zu machen.

Zunächst betrachten wir das Verhalten des feuchten Holzes*) bei tiefen Frequenzen (Abb. 6 und 7). Von einem gewissen Feuchtigkeitsgrad an steigen DK und $\operatorname{tg} \delta$ sehr stark mit fallender Frequenz an. Ein Dipoleffekt kommt hier

*) Es wird angenommen, da B im Darrzustande noch eine gewisse kleine Menge etwa $0,6 \%$ Wasser im Holze vorhanden ist. 
nicht in Frage, da die Relaxationszeiten für die beiden Komponenten - Zellsubstanz und Wasser - sehr klein sind. Vielmehr ziehen wir die Wagnersche Inhomogenitätstheorie ${ }^{16}$ ) zur Klärung heran. Sobald der Wassergehalt eine bestimmte Grenze überschritten hat, haben wir es mit einem Schichtkörper: Zellensubstanz + Wasser zu tun. Wir haben dann elementare Zweischichtkondensatoren, von denen die eine Schicht (Zellsubstanz) eine relativ kleine DK und schlechte Leitfähigkeit besitzt, während die andere (Wasser) eine sehr hohe DK hat. Diese Kombination kann sehr große Zeitkonstanten oder Relaxationszeiten ergeben, so da $B$ die Frequenz für $\operatorname{tg} \delta=$ Max. weit unter $10^{3} \mathrm{~Hz}$ liegen kann. Der Anstieg des $\operatorname{tg} \delta$ und der DK mit abfallender Frequenz wäre auf diese Weise zu erklären.

Betrachten wir weiter das Verhalten bei hohen Frequenzen (Abb. 3 und 4), so zeigt sich zunächst in dem Gebiet von $10^{5}$ bis $10^{6} \mathrm{~Hz}$ kein wesentlicher Einflu $B$ der Feuchtigkeit. Die im Schrifttum ${ }^{18}$ ) vorkommende Angabe, daß der Feuchtigkeitsgrad bei Holz und Papier bei hohen Frequenzen nur geringen Einfiuß auf den Verlustfaktor hat, bezieht sich anscheinend auf dieses Gebiet. Bei höheren Frequenzen tritt aber ein sehr wesentlicher Einfluß der Feuchtigkeit, soweit es sich um den tg $\delta$ handelt, auf. Bei der DK dagegen können wir nur eine Erhöhung mit steigendem Wassergehalt, aber im hohen Frequenzgebiet keine wesentlichen Abweichungen im Verlauf im Vergleich mit dem Darrzustande feststellen.

Man kann den Einfluß des Wassers nicht als einen reinen Mischvorgang deuten, bei dem an die Stelle der Luft im Gefüge des Naturholzes zum Teil Wasser mit seiner hohen DK tritt, denn dann müßte auch der Verlustfaktor und sein Verhalten aus dem EinfluB des Wassergehaltes, wie bei einer einfachen Mischung zu erklären sein. Nun hat aber Wasser eine sehr kleine Relaxationszeit und das Maximum von tg $\delta$ liegt erst etwa bei $1,48 \cdot 10^{10} \mathrm{~Hz}$ $(\lambda=1,8 \mathrm{~cm})$. Innerhalb des untersuchten Frequenzbereiches ist der $\operatorname{tg} \delta$ des Wassers klein und kann, auch wegen der relativ geringen Menge des Wassers, zur Klärung der Frhöhung des Verlustfaktors bei feuchtem Holz und der Verschiebung des Maximalwertes nicht herangezogen werden, wenn man von einem einfachen Mischdielektrikum ausgeht.

Es muB vielmehr, wenigstens im Bereich der untersuchten geringen Feuchtigkeitsgrade, der Einfluß der eindringenden Wassermoleküle auf die Dipolschwingungen in den Zellulosemolekülen als Ursache der zu diskutierenden Erscheinungen angesehen werden. Nach den im Schrifttum ${ }^{15}$ ) gegebenen Vorstellungen über den Mechanismus der Dipolschwingungen der Zellulose, beruhen sie hauptsächlich auf der freien Drehbarkeit der 3 Hydroxylgruppen, während die Sauerstoffbrücken zwischen den Glukoseresten starr bleiben (Abb. 9). Die Rechnungen, die Henniger ${ }^{8}$ ) durchgeführt hat, zeigen, daß das berechnete mittlere Moment bei Annahme einer freien Beweglichkeit der Hydroxylgruppen wesentlich über dem Moment liegt, das aus den experimentellen Werten abzuleiten ist. Er schlieBt daraus, daB die Beweglichkeit der Dipolgruppen bei der Zellulose stark behindert ist*).

Die Verschiebung des Maximalwertes des $\operatorname{tg} \delta$ nach höheren Frequenzen mit der Zunshme des Wassergehaltes würde bedeuten, daB die wahrschein-

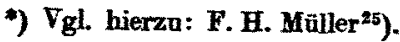


lichste Relaxationszeit $\tau_{0}$ in der Verteilungsfunktion mit wachsendem Wassergehalt abnimmt.

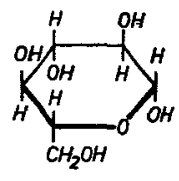

$\beta$-Glukose

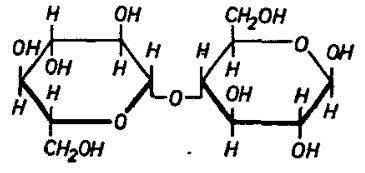

Zellabiose

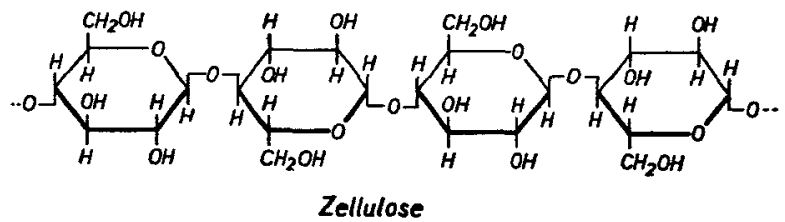

Abb. 9. Formeln der Zellulosereihe.

Betrachten wir den einfachsten Fall, bei dem die Rotation von kugelförmigen Dipolmolekülen mit dem Durchmesser $a$ in einer Flüssigkeit mit der inneren Viskosität $\eta$ für molekulare Dimensionen (,,innere Reibung") stattfindet, und zwar ohne wechselseitige Beeinflussung, so gilt die Debyesche Beziehung ${ }^{5}$ ), die auch annähernd für feste Körper bestehen bleibt,

$$
\tau=\frac{4 \pi \eta a^{3}}{k T}
$$

( $k$-Boltzmannsche Konstante; $T$-absolute Temperatur). Natürlich kann diese Beziehung nicht auf die komplizierten Verhältnisse der Zellulose übertragen werden, man kann sie aber anwenden, um bei Rückführung auf den Idealfall den Einfluß der einzelnen Parameter zu diskutieren.

Eine Vergrößerung der Relaxationszeit bei gleicher Temperatur und gleichen Molekülabmessungen würde durch eine Vergrößerung ron $\eta$ bedingt werden. $\mathrm{Da}$ nun nach den experimentellen Ergebnissen die wahrscheinlichste Relaxationszeit mit wachsendem Feuchtigkeitsgehalt kleiner wird, so zeigt das, daß die im trockenen Zustande vorhandene Hemmung der Bewegungen der Molekülgruppen durch das Wasser verkleinert wird. Abb. 10 und 11 zeigen nun nach Staudinger ${ }^{20}$ ) den Einfluß von Wasser auf mercerisierte Zellulose. In der $A b b .10$ sehen wir die parallelliegenden Fadenmoleküle durch Nebenvalenzen miteinander verbunden. Die Abb. 11 zeigt, wie durch die eindringenden Wassermoleküle die Querverbindungen gelockert werden. Es ist nun naheliegend, einen ähnlichen Vorgang auch bei der Zellsubstanz des Holzes anzunehmen, in dem man eine Lockerung der einzelnen parallelliegenden Makromoleküle durch das eindringende Wasser voraussetzt und daraus die Verschiebung der Relaxationszeiten nach kleineren Werten erklärt.

Es wäre nun von Interesse, festzustellen, wie sich die Bausteine der Zellulose, also die einzelnen $\beta$-Glukosemoleküle, dielektrisch verhalten. Die Abb. 12 stellt die von uns gemessene Abhängigkeit des $\operatorname{tg} \delta$ für ein Mischdielektrikum Glukose + Luft dar. Wir haben auch hier von einer Extrapolation der Werte für 
reine Glukose zunächst abgesehen, da, wie schon bei der Zellulose gezeigt und auch hier durch eine Abschätzung festgestellt wurde, durch die Luft-

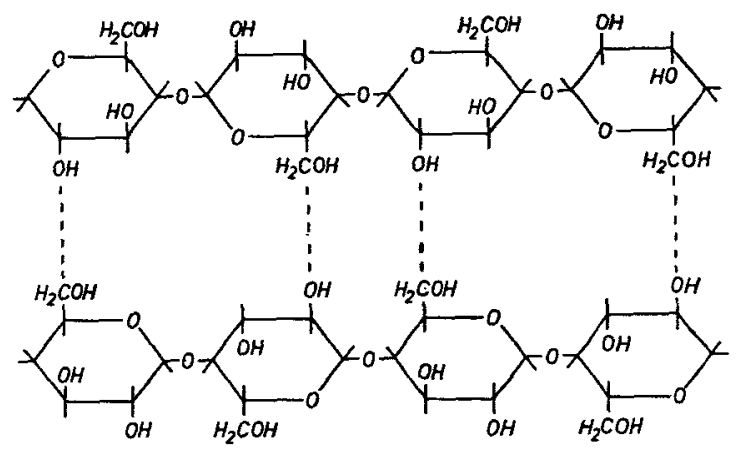

Abb. 10. Mercerisierte Zellulose: Ketten dureh Nebenvalenzen verbunden (nach Staudinger).

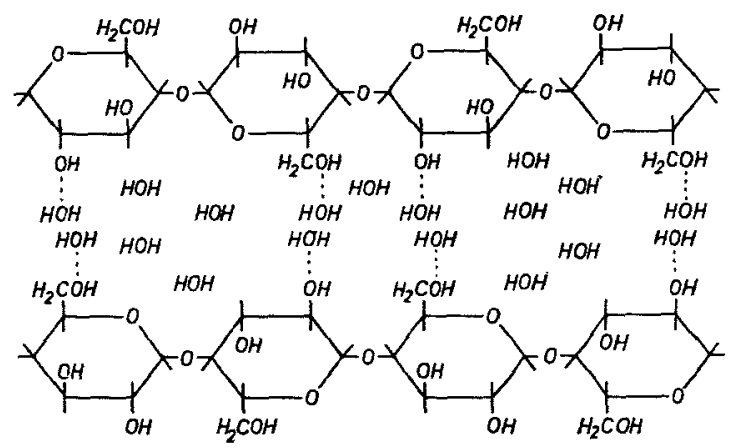

Abb. 11. Zelluloseketten mit Wasser gequollen, Bindung zwischen den Ketten aufgehoben (nach Staudinger).

beimischung die Frequenz $f_{0}$ und der Verlauf der Kurve in der Nähe des Maximums wenig beeinflußt werden. Wir erkennen, daß die Frequenz $f_{0}$ von $10^{7} \mathrm{~Hz}$ bei Zellulose, bei der Glukose anf $10^{8} \mathrm{~Hz}$ gestiegen, also eine Abnahme der Relaxationszeit eingetreten ist. Ferner ist die Kurve im Vergleich mit Abb. 7 wesentlich schmaler geworden. Wir müssen annehmen, daß die Hemmung der Dipolschwingungen gegenüber der Zellulose kleiner geworden ist. Die spitzere Kurve ist aus der jetzt vorhandenen Einheitlichkeit und Entkopplung der Dipolmoleküle zu erklären (vgl. S. 140 and Abb. 8).

Eine Cntersuchung, die vielleicht zum weiteren Studium des dielektrischen Verhaltens der Zellulose und damit auch der Holzstoffe beitragen könnte, wäre die Messung der Abhängigkeit von $\varepsilon^{\prime}$ und $\operatorname{tg} \delta$ von $f$ bei der Zellobiose, die aus 2 Glukoseresten aufgebaut ist (Abb. 9). Diese Messung konnten wir leider nicht durchführen, da die Beschaffung des Präparates schwierig war. Es ist uns auch unbekannt, ob dielektrische Untersuchungen an diesem Stoff 
im Schrifttum vorhanden sind. Natürlich müßten für weitere Behandlung der Frage die Werte für die reinen Stoffe aus den Messungen am Mischdielektrikum (z.B. durch eine Imprägnierungsmethode) ermittelt werden.

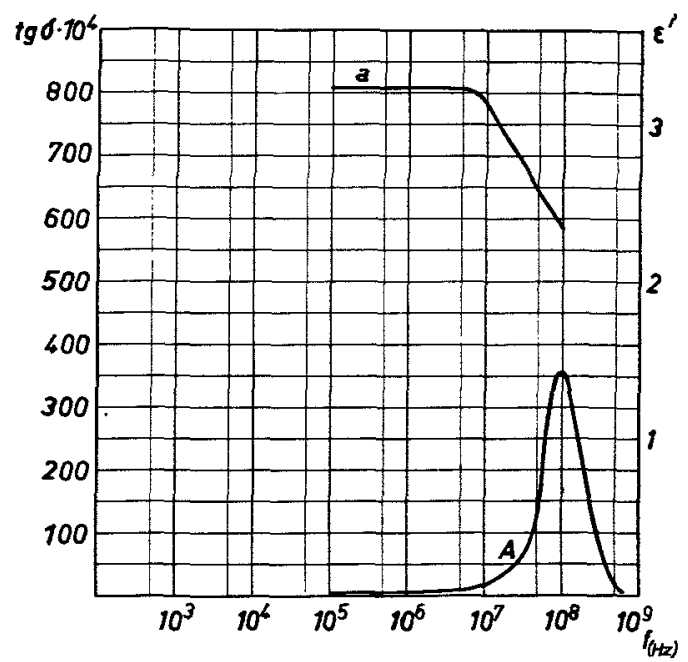

Abb. 12. $A-\operatorname{tg} \delta$ in Abhängigkeit von der Frequenz für Mischdielektrikum: Glukose $(55 \mathrm{Vol} \%)+$ Luft $(45 \%)$ bei 20 . $a-\mathrm{DK}$ füt reine Glukose.

Während die Relaxationszeit $\tau_{0}$ durch die Verkleinerung der inneren Reibung $\eta$ erniedrigt wird, wirkt nach der strengen Debyeschen Theorie die Verkleinerung der Kopplung mit den übrigen schwingenden Dipolgruppen der Umgebung im Sinne einer Vergrößerung von $\tau_{0}$. Bei experimentellen Untersuchungen wird diese Kopplung durch die Konzentration der Lösung eines Dipolstoffes in einer dipolfreien Flüssigkeit verändert. Beim Eindringen von Wasser müssen wir eine Verkleinerung der Kopplung gegenüber dem Trockenzustand annehmen. Dadurch würde aber unser Schluß in bezug auf die Änderung von $\tau_{0}$ unsicher. Es zeigt sich nun auf Grund von Untersuchungen von E. Fischer ${ }^{6}$ ) und W. Hacke17), daB die Debyesche Theorie nur für sog. ,normale Dipolfüssigkeiten“ gilt. Für andere Stoffe, wie z. B. Alkohole, tritt in bestimmten Frequenzgebieten ein umgekehrtes Verhalten ein, d.h. mit abnehmender Konzentration sinkt $\tau_{0}$ nach einem Maximum beträchtlich. Dieses Verhalten wird wie folgt gedeutet: Bei den normalen Dipolflüssigkeiten wird die Wirkung auf einen Dipol durch eine Anzahl ron Molekülen der näheren Umgebung bestimmt, wie es auch bei der Debyeschen Theorie angenommen wird. Bei den Alkoholen entsteht durch die polaren OH-Gruppen eine Wechselwirkung zwischen einem Molekül und seinen nächsten Nachbarn, die ein abweichendes Verhalten im Sinne einer mit der Konzentration wachsenden Relaxationszeit bewirken.

Nun kann bei der Zellulose ein āhnlicher Vorgang rermutet werden, bei dem eine Wechselwirkung mit den unmittelbaren Nachbarmolekülen über die 
oH-Gruppen erfolgt. Eine Einlagerung von Wassermolekülen würde sich dann ähnlich auswirken, wie eine Verringerung der Konzentration. Es wäre damit eine Herabsetzung der Relaxationszeit verbunden. Die beobachtete Verringerung von $\tau_{0}$ bei Wassergehalt könnte dann entweder auf eine Verkleinerung der inneren Reibung oder eine Verringerung der Wechselwirkung der Dipole zurückgeführt werden. Welche Wirkung die entscheidende ist, läßt sich noch nicht sagen.

\section{Zusammenfassung}

Es wird eine frühere Arbeit, die sich auf das Verhalten der DK bei Naturholz im großen Frequenzbereich $3 \cdot 10^{2} \ldots 1 \cdot 10^{9} \mathrm{~Hz}$ bezog, auf den Verlustfaktor erweitert. Es wird, ähnlich wie bei der DK, auch hier eine Anisotropie bei verschiedenen Schnitten und eine Dispersion mit der Frequenz festgestellt. Es tritt ein breites Maximum des Verlustfaktors in der Nähe der Frequenz $10^{7} \mathrm{~Hz}$ auf. Die Versuche werden auf feuchtes Holz ausgedehnt. Es wird hier eine Verschiebung des Maximums des Verlustfaktors nach höheren Frequenzen und eine Erniedrigung des Wertes von $\tau_{0}$ beobachtet.

Die Deutung der Anisotropie des Verlustfaktors erfolgt, wie auch bei der DK, durch die makroskopische Struktur des Naturholzes. Das Auftreten eines Maximums für eine bestimmte Frequenz wird aus den polaren Eigenschaften der Zellsubstanz erklärt. Die Verschiebung dieses Maximums zu höheren Frequenzen bei feuchtem $\mathrm{Holz}$ wird durch eine Wassereinlagerung zwischen den Molekülketten der Zellulose gedeutet. Für tiefe Frequenzen ist bei Wassergehalt des Holzes ein Inhomogenitätseffekt im Sinne der Wagnerschen Theorie wahrscheinlich.

Wir danken den Herren Dr. Klauditz und Dr. Luther für wertvolle Diskussionen, ferner den Herren Dipl.-Ing. W. Trapp, H. Rummert und Wolfgang Pungs für freundliche Mitarbeit. Zu ganz besonderem Dank sind wir der Braunschweigischen Wissenschaftlichen Gesellschaft verpflichtet, die durch finanzielle Unterstützung die Fortführung dieser Untersuchungen ermöglichte.

Institut für Fernmelde- und Hochirequenztechnik der T. H. Braunschweig 


\section{Literaturverzeichnis}

1) Bätz, O., Über Verlustmessungen an Dipolflüssigkeiten und festen techn. Isolierstoffen bei Zentimeterwellen, Phys. Zeitschrift 1939, S. 394.

2) Böning, P., Elektrische Isolierstoffe, Verl. Friedr. Vieweg \& Sohn, Braunschweig.

3) Brake, E., u. H. Schütze, ENT 12 (1935), S. 120.

4) Büchner, A., Das Mischkörperproblem in der Kondensatortechnik, W. V.a.d. Siemens-Werken, Bd. 18, 2. H. (1939), S. 84.

5) Debye, P., Polare Molekeln, Verlag S. Hirsch, Leipzig 1929.

6) Fischer, E., Dielektrische Relaxationsuntersuchungen im Hinblick auf die molekulare und intermolekulare Struktur von Dipolflüssigkeiten, Phys. Zeitschrift 1939, Nr. 21, S. 645 .

7) Hackel, Die Temperaturabhängigkeit der Dipolleitfähigkeit von Alkoholen, Phys. Zeitschrift 1937, S. 195.

8) Henninger, Dielektrische Untersuchungen an der Papierfaser, Frequenz 4 (1950), S. 167.

9) Henninger, Dielektrische Untersuchungen an flüssigen Isolierstoffen mit Polarkomponente; Frequenz 4 (1950), S. 233.

10) Holzmüller, Beitrag zur Theorie der anomalen Dispersion und der dielektrischen Verluste in Festkörpern, Phys. Z. 41 (1940), S. 409.

11) Holzmüller, Dielektrische Verluste hochpolymerer Festkörper, Phys. Zeitschrift 42 (1941), Nr. 15/16, S. 287.

12) Kröner, Dissertation Braunschweig, 1943.

13) Lindmann, K. F., Über die Doppelbrechung elektrischer Wellen in Eichenholz, Z. f. techn. Phys. Nr. 3 (1939), S. 72.

14) Müller, F. H., Physik des organischen Isolators, ETZ 59 (1938), S. 1155.

15) Müller, F. H., u. Chr. Schmelzer, Dielektrisches Verhalten im Zusammenhang mit dem polaren Aufbau der Materie. Ergebnisse der exakten Naturwissenschaften 25 (1951), S. 359 (dort auch neuestes Schrifttum bis 1951).

16) Schering, H., Zusammenfassende Darstellung der Wagnerschen Theorie, in: „Die Isolierstoife der Elektrotechnik".

17) Sharpe, B. A., u. B. J. O'Kane, Engineering 140 (1935), S. 403.

18) Schupp, P. O., Zur Physik der dielektr. Verluste, W. V.a.d. Siemens-Konzern XVII, H. 1 (1938).

19) Schweidler, Ann. d. Phys. 24 (1907), 711.

20) Staudinger, H., Organische Kolloidchemie, Verl. Friedr. Vieweg \& Sohn, Braunschweig, 1950.

${ }^{21}$ ) Sta udinger, H., Über hochpolymere Verbindungen, Z. Holz als Roh- und Werkstoff, 1938 , H. 7.

${ }^{22}$ ) Stoops, W. N., The dielectr. properties of cellulose, Proc. and Journ. of the american. chem. Sec. 56 (1934), S. 1481.

23) Wagner, K. W., Zur Theorie der unvollkommenen Dielektrika, Ann. d. Phys. IV, Bd. 40 (1913), S. 817.

24) Yagar, W. A., The distribution of relaxation times in typical dielectrics. Physics 7 (1936), S. 431 .

25) F. H. Müller, Kunststoffe 41 (1951) S. 280. 